\title{
Hot-electron nanoscopy using adiabatic compression of surface plasmons
}

\author{
A. Giugni1,2, B. Torre ${ }^{2,3}$, A. Toma', M. Francardi2,4, M. Malerba', A. Alabastri1, R. Proietti Zaccaria1, \\ M. I. Stockman $5,6,7$ and E. Di Fabrizio $2,4 \star$
}

\begin{abstract}
Surface plasmon polaritons are a central concept in nanoplasmonics and have been exploited to develop ultrasensitive chemical detection platforms, as well as imaging and spectroscopic techniques at the nanoscale. Surface plasmons can decay to form highly energetic (or hot) electrons in a process that is usually thought to be parasitic for applications, because it limits the lifetime and propagation length of surface plasmons and therefore has an adverse influence on the functionality of nanoplasmonic devices. Recently, however, it has been shown that hot electrons produced by surface plasmon decay can be harnessed to produce useful work in photodetection, catalysis and solar energy conversion. Nevertheless, the surface-plasmon-to-hot-electron conversion efficiency has been below $1 \%$ in all cases. Here we show that adiabatic focusing of surface plasmons on a Schottky diode-terminated tapered tip of nanoscale dimensions allows for a plasmon-to-hot-electron conversion efficiency of $\sim 30 \%$. We further demonstrate that, with such high efficiency, hot electrons can be used for a new nanoscopy technique based on an atomic force microscopy set-up. We show that this hot-electron nanoscopy preserves the chemical sensitivity of the scanned surface and has a spatial resolution below $50 \mathrm{~nm}$, with margins for improvement.
\end{abstract}

T he coupling of electromagnetic waves and electrons at the surface of a metal produces surface plasmon polaritons (SPPs). As a result of their intrinsic electromechanical nature ${ }^{1}$, SPPs can overcome both the optical diffraction limit and the mean free path of electrons in metals. SPPs provide an effective way to guide, localize and concentrate energy at the nanoscale ${ }^{2-7}$, offering the possibility to control fundamental energy transfer processes. It has been reported previously that hot electrons, highly energetic electrons created by the decay of surface plasmons, can generate a photocurrent in Schottky diodes composed of plasmonic nanoantennas supported on a semiconductor surface ${ }^{8,9}$. However, the quantum efficiency of the conversion of surface plasmons to electrons for photocurrent generation is much lower than in conventional photoexcited $\mathrm{p}-\mathrm{n}$ diode cells. This is due to the intrinsic suppression of the Schottky current imposed by linear momentum conservation for smooth Schottky contacts.

This suppression can be drastically reduced if the surface of the Schottky diode is rough, leading to a higher quantum efficiency for the plasmon-to-photocurrent conversion ${ }^{10}$. Recently, a plasmonic solar water splitter ${ }^{11}$, where hot electrons play a crucial role, has been demonstrated, and the fact that the surface was nanostructured improved the water splitting efficiency substantially although hot electron generation remained low, at below $1 \%$.

In this Article we report a surface plasmon-to-hot electron conversion efficiency of $\sim 30 \%$ at three different wavelengths. In particular, we generate a Schottky current through adiabatic compression of plasmons in a nanocone $e^{12,13}$ set in contact with the semiconductor, and we take advantage of this highly efficient conversion to introduce a novel scanning nanoscopy technique.
Because the Schottky current is inherently sensitive to the difference in Fermi energies between the metal of the nanocone and the semiconductor surface, our nanoscopy set-up is sensitive to the type of semiconductor, its doping and surface impurities. A significant advantage of using an adiabatic concentration of SPPs ${ }^{14-16}$ in the Schottky configuration is the decay rate of the SPPs, which is inversely proportional to the taper radius $R$ of the plasmonic nanocone ${ }^{15}$ when $R$ is less than or equal to the plasmonic skin depth, which in the case of gold is $\sim 25 \mathrm{~nm}$ (see equations in Supplementary Section 8). A tapered waveguide, because of its favourable conditions for high energy concentration and broad wavevector and momentum exchange, efficiently transforms a propagating SPP wave into a quasi-static local field and into hot electrons. This is the underlying principle for the high conversion efficiency of the proposed nanoscopy.

We performed three different experiments to prove the hot electron contribution in the Schottky junction configuration. In the first, we measured the maximal photocurrent generated by the SPPs using gold as metal and GaAs as a prototype semiconductor sample, both through photovoltaic and purely hot electron conversion. In the second, we measured the $I-V$ curves of the Schottky diode in the dark and for three wavelengths above and below the $\mathrm{GaAs}$ bandgap $\left(E_{\text {gap }} \approx 1.45 \mathrm{eV}\right)$. In the third experiment we used the SPP-generated photocurrent to image either a locally patterned oxidized or an ion-implanted conductive GaAs surface as a proof-of-concept demonstration of scanning probe nanoscopy based on hot electrons.

Plasmonic concentrator set-up

Recently, it has been shown that SPPs can propagate to and concentrate adiabatically at the apex of tapered plasmonic waveguides ${ }^{15-18}$, thus

\footnotetext{
'Nanostructures, Istituto Italiano di Tecnologia, via Morego 30, 16163 Genova, Italy, ${ }^{2}$ King Abdullah University of Science and Technology, PSE and BESE Divisions, Thuwal, 23955-6900, Kingdom of Saudi Arabia, ${ }^{3}$ Nanophysics, Istituto Italiano di Tecnologia, Via Morego 30, 16163 Genova, Italy, ${ }^{4}$ BIONEM, Bio-Nanotechnology and Engineering for Medicine, Department of Experimental and Clinical Medicine, University of Magna Graecia Viale Europa, Germaneto, 88100 Catanzaro, Italy, ${ }^{5}$ Max-Planck-Institut für Quantenoptik, Hans-Kopfermann-Strasse 1, D-85748 Garching, Germany, ${ }^{6}$ Department of Physics, Georgia State University, Atlanta, Georgia 30340, USA, ${ }^{7}$ Fakultät für Physik, Ludwig-Maximilians-Universität, Geschwister-Scholl-Platz 1 , D-80539 München, Germany. *e-mail: enzo.difabrizio@kaust.edu.sa
} 

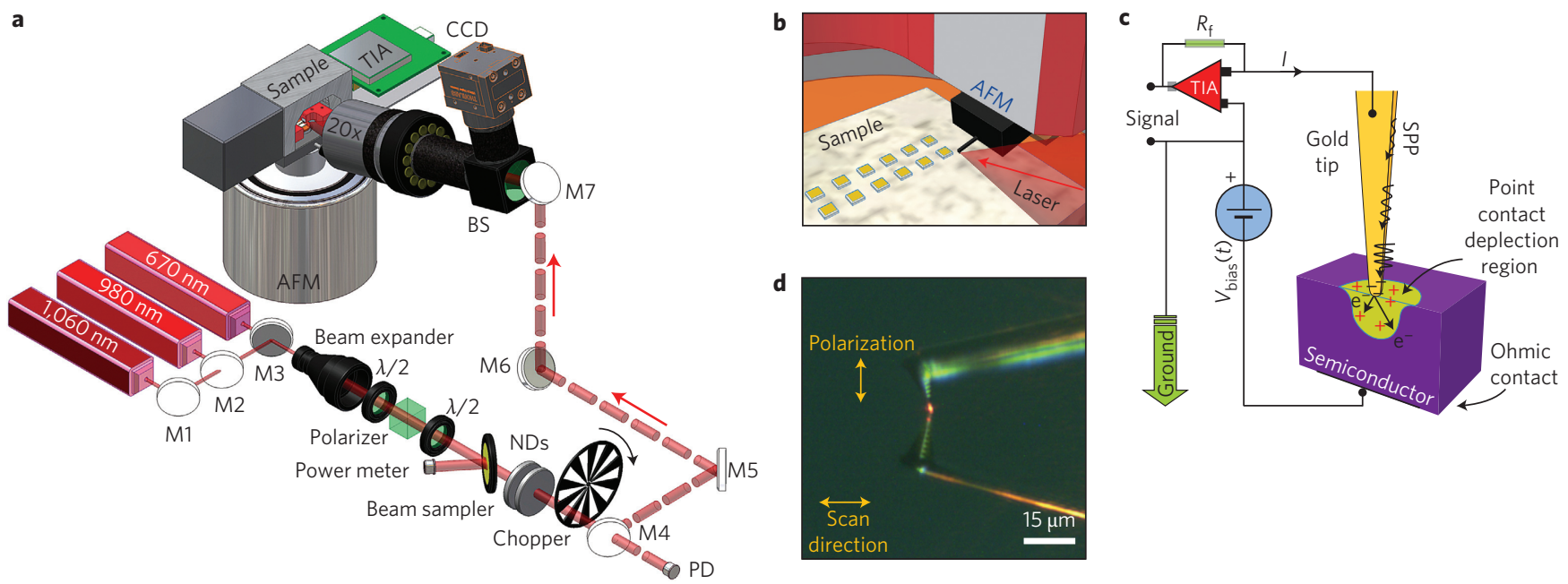

Figure 1 | Schematic of experimental set-up. a, Complete optical layout. Vertically polarized continuous-wave laser excitation at 670 nm, 980 nm or $1,060 \mathrm{~nm}$ ( $\sim 10 \mu \mathrm{W}$ of optical power on the coupling grating) was amplitude-modulated by a mechanical chopper wheel at $\sim 500 \mathrm{~Hz}$ and focused on the structured AFM tip. Additional elements, as indicated, were used to monitor (photodiode, PD) and manage the polarization, intensity (neutral density filters, NDs) and beam profile of the incident radiation. M1-M7 are mirrors; BS, beamsplitter. b. Close-up sketch of the sample configuration. c, Basic circuit scheme: a transimpedance amplifier (TIA) FEMTO, gain $=10^{8}$, bandwidth $=50 \mathrm{kHz}$ performed the junction current amplification. An external bias voltage circuit was used to polarize the junction, and a lock-in amplifier SR830 and a NI6366 data acquisition board were used to acquire and record the signals. $\mathbf{d}$, A confocal CCD (charge-coupled device) allows real-time monitoring of the scanning and illumination states. The optical spot at the cone apex is a result of the radiative decay of the SPP when the device is illuminated by a laser with a wavelength of $670 \mathrm{~nm}$. One of the twin images is the reflection from the GaAs surface. For further details on additional components see Supplementary Section 3.

offering the potential to guide and localize optical energy with acceptable losses. Furthermore, by appropriately tuning the surface roughness and structure geometry, it is possible to efficiently promote specific SPP damping channels ${ }^{19-24}$, moving from radiative $\operatorname{losses}^{25,26}$ to the generation of hot electrons as a primary decay channel.

To quantify the contribution of hot electrons in the detectable current, we developed an atomic force microscope (AFM)based plasmonic concentrator that allows mapping of the photoelectric signal with nanoscale lateral resolution (in the same length range as the tip contact diameter) and a spectral bandwidth limited only by the high-gain current amplifier. All measurements were performed in contact mode. The set-up, illustrated schematically in Fig. 1 and Supplementary Fig. 8, consists of an AFM customized with a high gain bandwidth $($ GBW $)$ trans-impedance current amplifier $\left(\mathrm{GBW} \approx 1 \times 10^{13}\right)$ and an optical layout adequate to properly promote the adiabatic SPP compression. The present set-up allows the morphological profile and the current generated across the Schottky barrier to be recorded simultaneously.

To estimate the plasmonic performances of our device we simulated the full three-dimensional structure (grating + cone) of Fig. 2a, as shown in Fig. 2b (see Methods). The chosen metal was gold, and the surrounding medium was air. Numerical calculations were performed at different wavelengths. Figure $2 \mathrm{~b}$ shows the results for infrared radiation $(\lambda=1,060 \mathrm{~nm})$ and for an incidence angle of $\theta \approx 36.5^{\circ}$ with respect to the normal of the surface (as defined by the geometry of the optical set-up). The AFM tip has a pyramidal shape with an octagonal base (height $15 \mu \mathrm{m}$ ) and $40^{\circ}$ full-angle aperture, and the height and base of the cone are $2.5 \mu \mathrm{m}$ and $300 \mathrm{~nm}$, respectively. The tip radius was set to $25 \mathrm{~nm}$, equal to the measured value. Details about the numerical simulation are given in Supplementary Section 1.

In Supplementary Section 11 we report additional experimental results for cantilevers without engraved gratings, as well as for cantilevers with chromium as a non-plasmonic metal coating. We performed these controls to further elucidate the role of the material, the illumination geometry and the device architecture ${ }^{27,28}$.

\section{Photocurrent measurement}

The dominant effect in our device was enhancement of the internal photoemission (IPE; for details on other terms contributing to the $I-V$ characteristic see Supplementary Section 7). It is known that the point contact geometry at the nanoscale strongly departs from the one-dimensional Schottky barrier model ${ }^{29,30}$ (Supplementary Section 6), even for moderately doped semiconductors. The localization of the photoconversion process close to the contact leads to a specific $I-V$ characteristic that reflects the local electronic structure on the spatial length scale of the contact. In a rather simplified picture, the unique characteristic of a Schottky contact, compared to the classical $\mathrm{p}-\mathrm{n}$ junction, is that the photocurrent can be generated by the direct electromagnetic field absorption in the metallic active layer-the IPE process. This can also happen when the SPP quantum energy exceeds the Schottky barrier height, $h v \geq e \Phi_{\mathrm{b}}$, where $e$ is the electron charge (Fig. 2c). In particular, when the excited electrons at the interface pass over the potential barrier, provided they have enough energy and eventually the appropriate momentum, they enter the depletion region of the semiconductor and then relax to lower states of the conduction band. These electrons can eventually be collected as a photocurrent, even with no reverse bias (electrons move from tip to semiconductor) or a direct bias voltage $V_{\text {bias }}<h v-e \Phi_{\mathrm{b}}$. The optimal thickness of the metallic layer, in terms of IPE current production, is a tradeoff between the number of excitable electrons, which is proportional to the thickness of the metallic layer, and the electron mean free path in the metal itself, typically a few tens of nanometres for gold $^{31,32}$. Normally, the collection efficiency of electrons in the Schottky photodiodes is severely limited by the availability of sufficient kinetic energy in the direction normal to the interface. This efficiency generally corresponds to $\sim 1 \%$ of the typical photovoltaic contribution, thus hindering the practical utility of the present system as a photovoltaic cell. Nevertheless, Schottky 

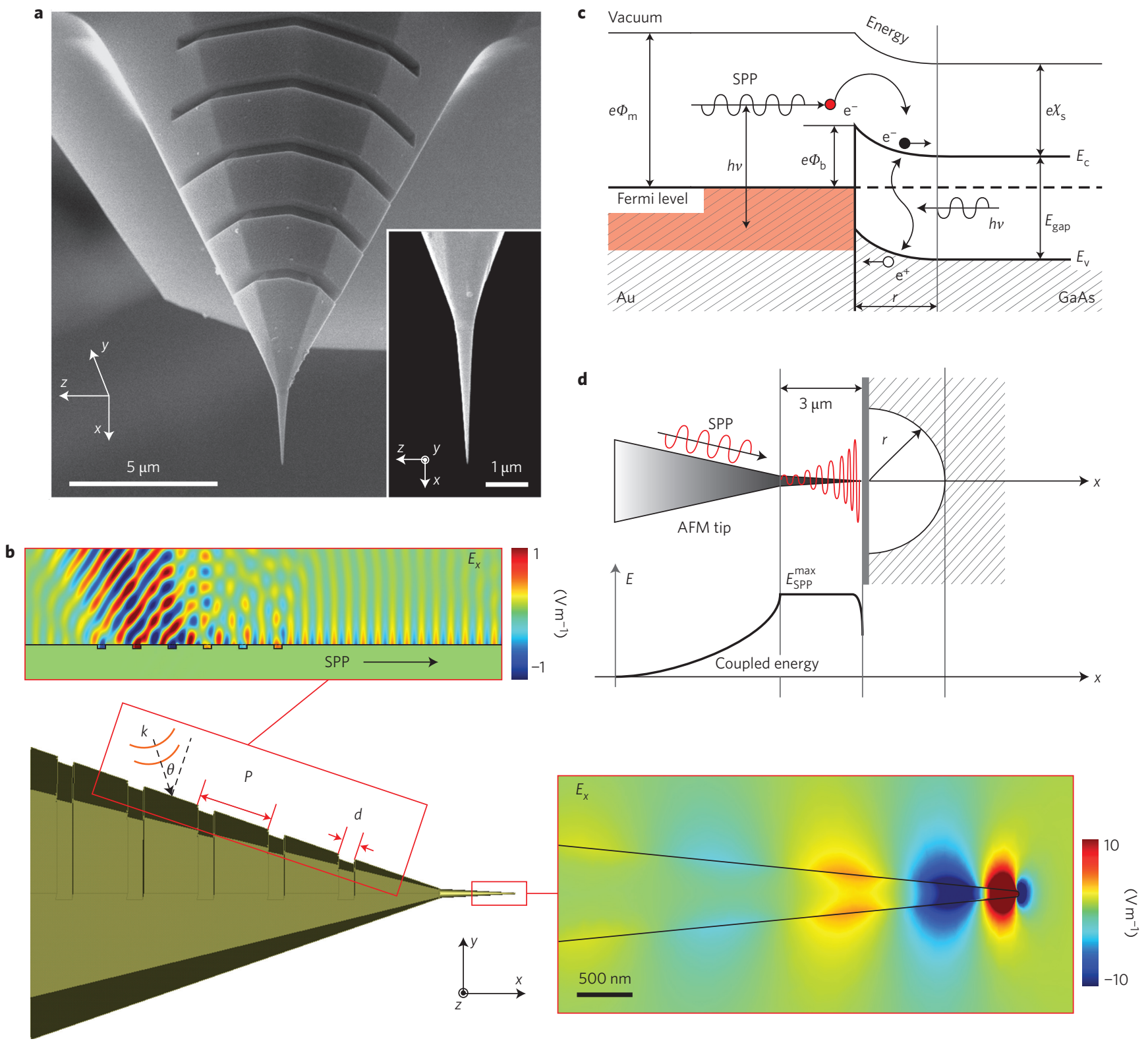

Figure 2 | Plasmonic structure and scheme of SPP generation. a, SEM images of grating and cone fabricated on an octagonal pyramid. $\mathbf{b}$, Grating parameters at $\lambda=1,060 \mathrm{~nm}$; pitch $P=2.43 \mu \mathrm{m}$; groove size $d=365 \mathrm{~nm}$; groove depth $h=140 \mathrm{~nm}$. The cone height is $2.5 \mu \mathrm{m}$ and the base diameter is $B=300 \mathrm{~nm}$. A fully $3 \mathrm{D}$ simulation was used for calculating the coupling efficiency of the laser with grating. For clarity, we reported in the insets the 2D projection of the $x$-component of the electric field amplitude along the grating and at the tip apex (xy plane). c, Band diagram at the metal/semiconductor interface showing the energy of the hot electrons (generated by SPP decay) with respect to the Fermi level of the metal and the energy of the semiconductor gap, $E_{\text {gap }}$ in the case of $h v \geq E_{\text {gap. }} \Phi_{\mathrm{m}}$ and $\chi_{\mathrm{s}}$ are the metal workfunction and semiconductor susceptivity, respectively, $r$ is the depletion radius, and $E_{\mathrm{c}}$ and $E_{\mathrm{v}}$ are the energy of the conduction band edge and valence band edge, respectively. $\mathbf{d}$, Scheme of SPP generation at the tip junction Au/GaAs interface. The hemispherical depletion region is generated by the point contact configuration of the cone apex. 'Coupled energy' refers to the laser energy fraction that the grating delivers to the nanocone as SPPs for hot electron generation.

photodetectors are appreciated for their fast response due to their one-carrier current character as well as for their low photonenergy detection capability 33 .

Photoexcitation was performed at three laser wavelengths: $\lambda_{1}=670 \mathrm{~nm} \quad\left(1.85 \mathrm{eV}>E_{\text {gap }}\right), \quad \lambda_{2}=980 \mathrm{~nm} \quad\left(E_{\text {gap }}>1.26 \mathrm{eV}>\right.$ $\left.e \Phi_{\mathrm{b}}\right)$ and $\lambda_{3}=1,060 \mathrm{~nm}\left(E_{\text {gap }}>1.17 \mathrm{eV}>e \Phi_{\mathrm{b}}\right)$, s-polarized in the normal plane of the grating grooves (Supplementary Fig. 8c). For GaAs illuminated at $\lambda_{2}$ and $\lambda_{3}$, only the IPE process contributes to the detected photocurrent, because direct photoabsorption in the semiconductor (photovoltaic contribution) is not energetically allowed. To improve the sensitivity we modulated the laser amplitude on-off with a square wave with a frequency that was lower than the typical thermalization timescale in semiconductors. The tip-to-sample current, under illumination and in dark conditions ( $I_{\text {on }}$ and $I_{\text {off }}$, respectively), was obtained either via lock-in detection or retrieved via post-processing software analysis (for details about the data acquisition and subsequent analysis procedure see Supplementary Section 3). In Fig. 3a-c we report a representative set of tip-to-sample current temporal profiles (GaAs-Au tip junction illuminated at $\lambda_{1}, \lambda_{2}$ and $\lambda_{3}$ ), together with the synchronism signal of the laser pulses. Each temporal sequence clearly reveals the SPP photoinduced nature of the measured signal. In Fig. 3d we also report the cantilever deflection signal in 'on' and 'off' states as a check on the temperature dependence 

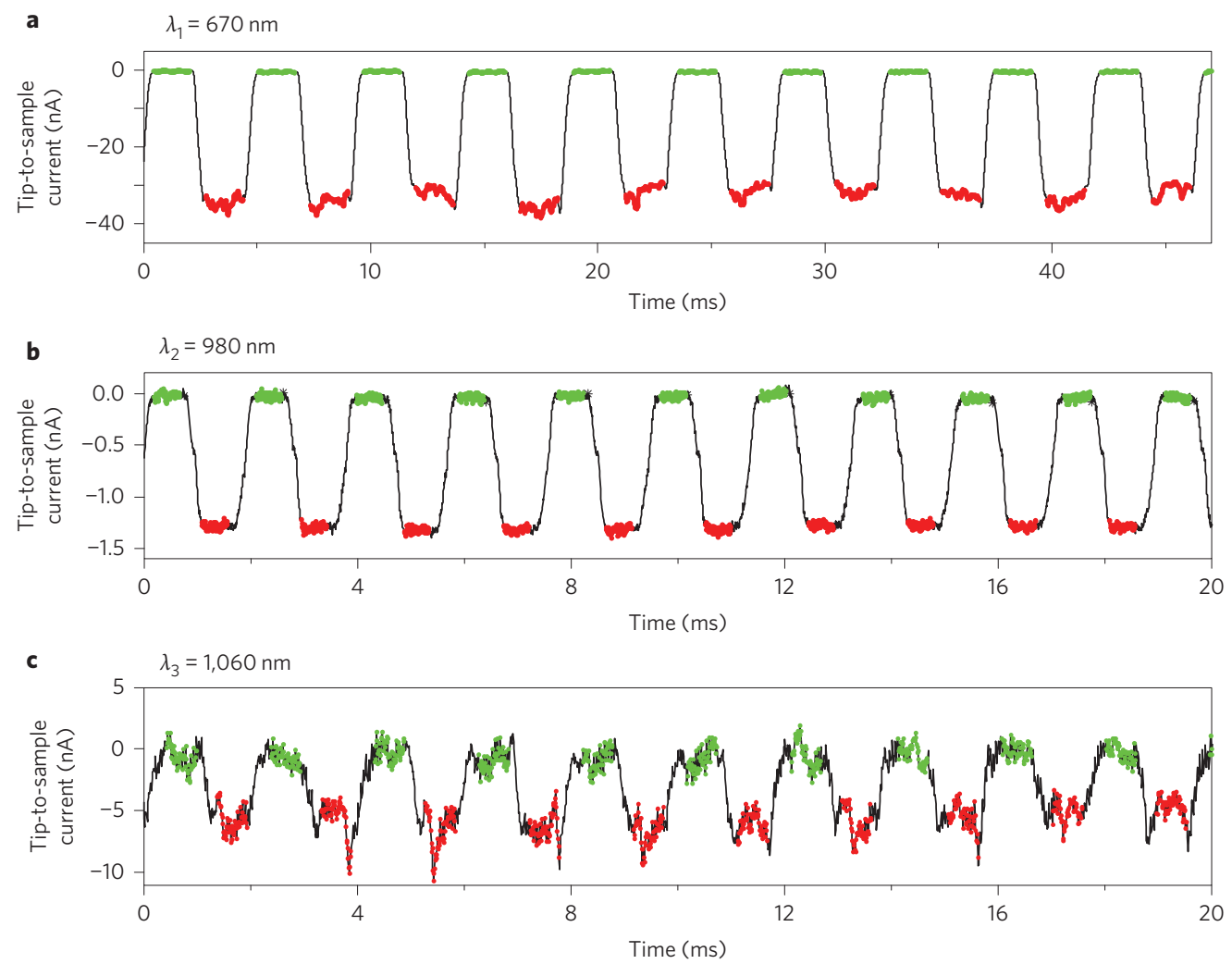

d

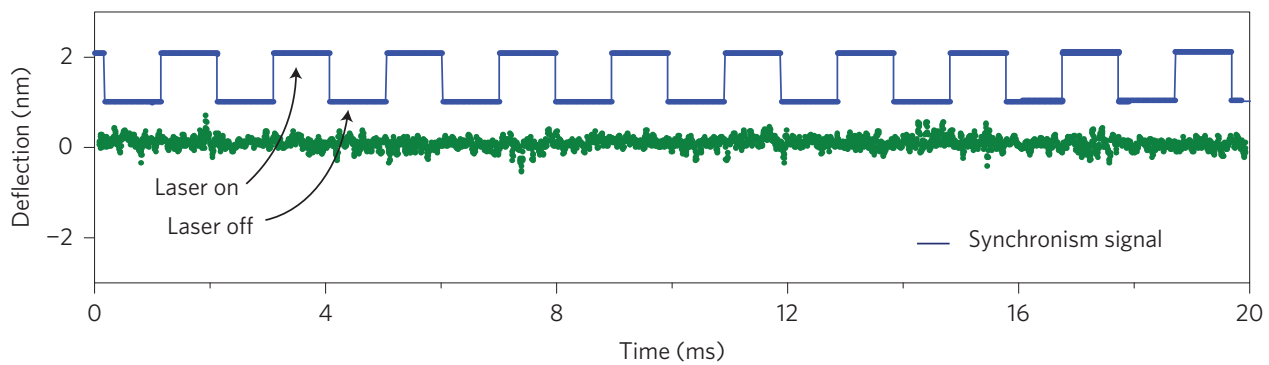

Figure 3 | Photoelectric AFM current through the n-type GaAs-Au tip junction. a-c, $I_{\text {on }}$ and $I_{\text {off }}$ current measurements through the $n$-type GaAs-Au tip junction for a fixed bias value of $-2 \mathrm{~V}$ at three laser wavelengths $\lambda_{1}, \lambda_{2}$ and $\lambda_{3}$, with impinging laser power on the grating of 10,4 and $10 \mu \mathrm{W}$, respectively. Red and green coloured data were successively mediated to obtain the $I-V$ characteristic for the on and off conditions, respectively. $\mathbf{d}$, Temporal profile of the cantilever deflection signal and of the laser modulation signal.

a

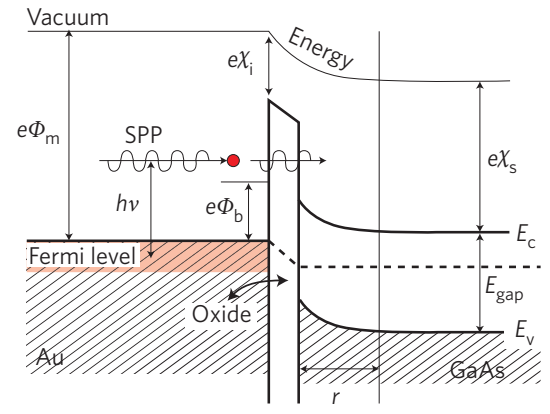

b

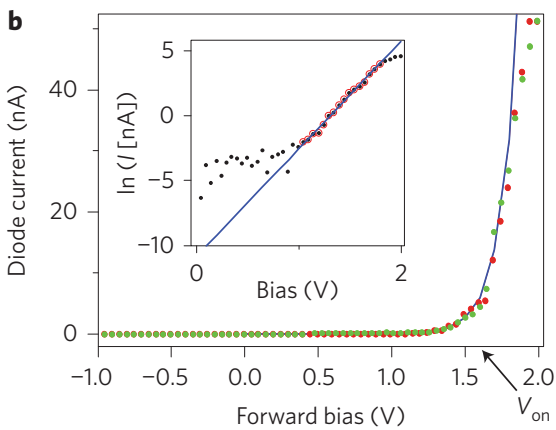

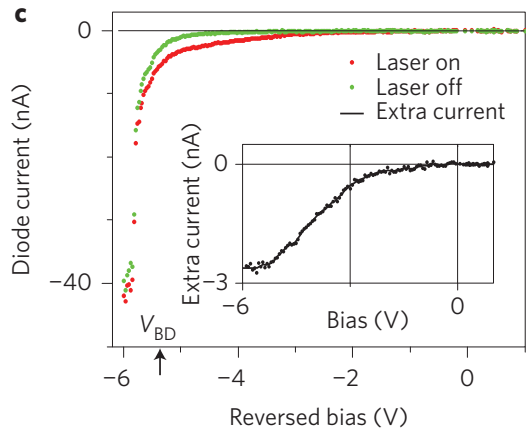

Figure $4 \mid$ I-V characteristics of the nanosized Schottky junction. a, Energy band diagram of the Au-GaAs junction with an oxide layer ( $0.7 \mathrm{~nm}$ thick; $\chi_{\mathrm{i}}$ is the insulator susceptibility). This band scheme is compatible with the extra current value obtained by the $I-V$ characteristic of the tip junction. $\mathbf{b}, \mathbf{c}$, Forward (b) and reverse (c) bias on (red) and off (green) I-V characteristics of the nanosized Schottky junction at a radiation wavelength of 1,060 nm. In the inset in b (on log scale), the blue line is the thermionic emission diffusion model used to fit to experimental $I_{\text {off }}$ data, and $V_{\text {on }}$ is the onset voltage of the Schottky diode indicating the ohmic conduction region. In $\mathbf{c}$, the inset shows the extra current defined as $I_{\text {on }}-I_{\text {off }}(n A)$, that is, the hot-electron net current. Note the early breakdown voltage $V_{\mathrm{BD}}$ value at $\sim 5.5 \mathrm{~V}$. 

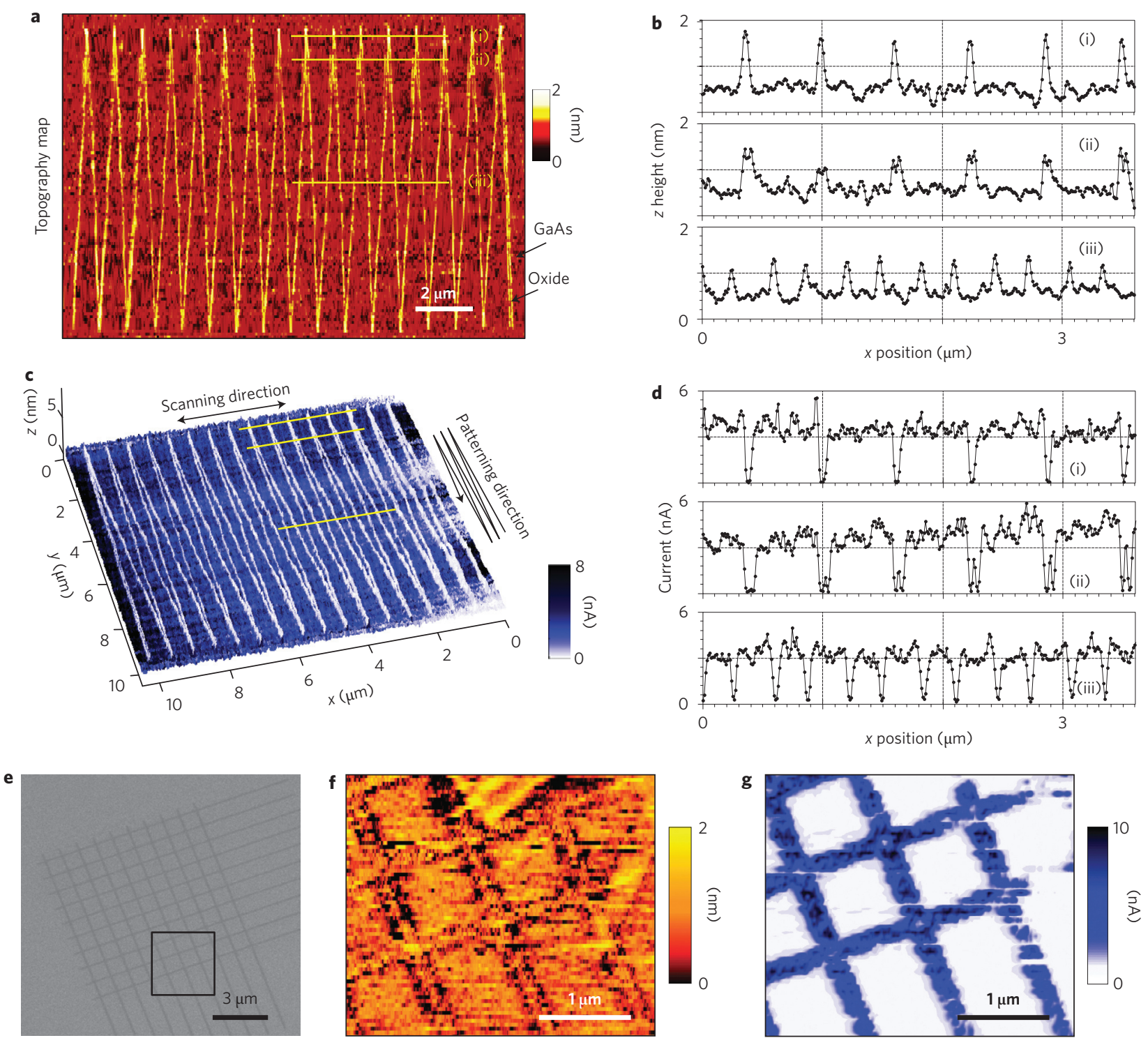

Figure 5 | Three-dimensional hot-electron maps of specific custom-realized locally patterned samples. Topography and photocurrent maps show both locally oxidized surfaces and ion-implanted conductive samples, respectively excited at $\lambda_{1}$ and $\lambda_{2}$. a,b. High-resolution AFM topography and height profiles of a continuous oxide pattern deposited on GaAs made by a top-down fabrication technique through high field discharge in water ( $\sim 40 \%$ ambient air humidity). The pattern was written using the same plasmonic tip with $+4 \mathrm{~V}$ sample bias at $4 \mu \mathrm{m} \mathrm{s}{ }^{-1}$ writing speed, in contact mode (set point $10 \mathrm{nN}$ ). Topography map and profiles (indicated by yellow lines in the map) are not deconvolved for the tip profile. c, Photocurrent imaging overlaid on three-dimensional topography, showing simultaneously the achieved current and topographic resolution. $\mathbf{d}$, Single line photocurrent intensity profiles indicated with a yellow line on image $\mathbf{c}$. The photocurrent measure was performed by scanning in AFM contact mode with a $90^{\circ}$ angle to the patterned surface, under a $\mathrm{N}_{2}$ atmosphere. The zigzag profile allows a direct check of experimental resolutions from line profiles (ii) of $\mathbf{b}$ and $\mathbf{d}$. e, SEM image of Ga ionimplanted GaAs sample. f,g, Topography and plasmonic hot-electron maps, generated at 980-nm laser excitation, acquired in the region indicated by a black rectangle in $\mathbf{e}$. The pattern was fabricated by a focused ion beam process as single grid lines (40 pA, $100 \mathrm{~ns} \mathrm{point}^{-1}, 30 \mathrm{keV}$, single pass).

induced by the illumination and its hot electron current generation. In Supplementary Section 9 we simulate the dependence of temperature on laser illumination. The results show that the temperature increase is negligible (less than $10 \mathrm{~K}$ ) at our illumination power.

\section{Characteristics of the tip junction Schottky barrier}

To elucidate the hot electron contribution to the photocurrent (with respect to the photovoltaic contribution), we measured several $I-V$ characteristics of the n-type GaAs-Au tip junction between the breakdown and conduction regime (typically in the bias range between $-6 \mathrm{~V}$ and $+4 \mathrm{~V}$ ). Figure 4 shows representative $I-V$ characteristics of the Schottky diode with the laser (wavelength $\lambda_{3}$ ) respectively on and off (corresponding $I-V$ curves at $\lambda_{1}$ and $\lambda_{2}$ are reported in Supplementary Section 4).

Based on the thermionic emission, the $I-V$ characteristics of the Schottky barrier, for a diode operated at $V>3 k T / q$, are described by the equation ${ }^{33,34}$

$$
I_{\mathrm{F}}=S \cdot A^{* *} T^{2} \exp \left(\frac{q \Phi_{\mathrm{B} 0}}{K T}\right) \exp \left(\frac{q\left(V-I_{\mathrm{F}} R_{\mathrm{S}}\right)}{n K T}\right)
$$

where $I_{\mathrm{F}}$ is the forward current, $S$ is the area of the gold contact, $A^{* *}$ is the effective Richardson constant $\left(8 \mathrm{~A} \mathrm{~cm}^{-2} \mathrm{~K}^{-2}\right.$ for our n-type GaAs; ref. 35$), T$ is the absolute temperature, $K$ is the Boltzmann constant, $q$ is the electron charge, $\Phi_{\mathrm{B} 0}$ is the barrier height 

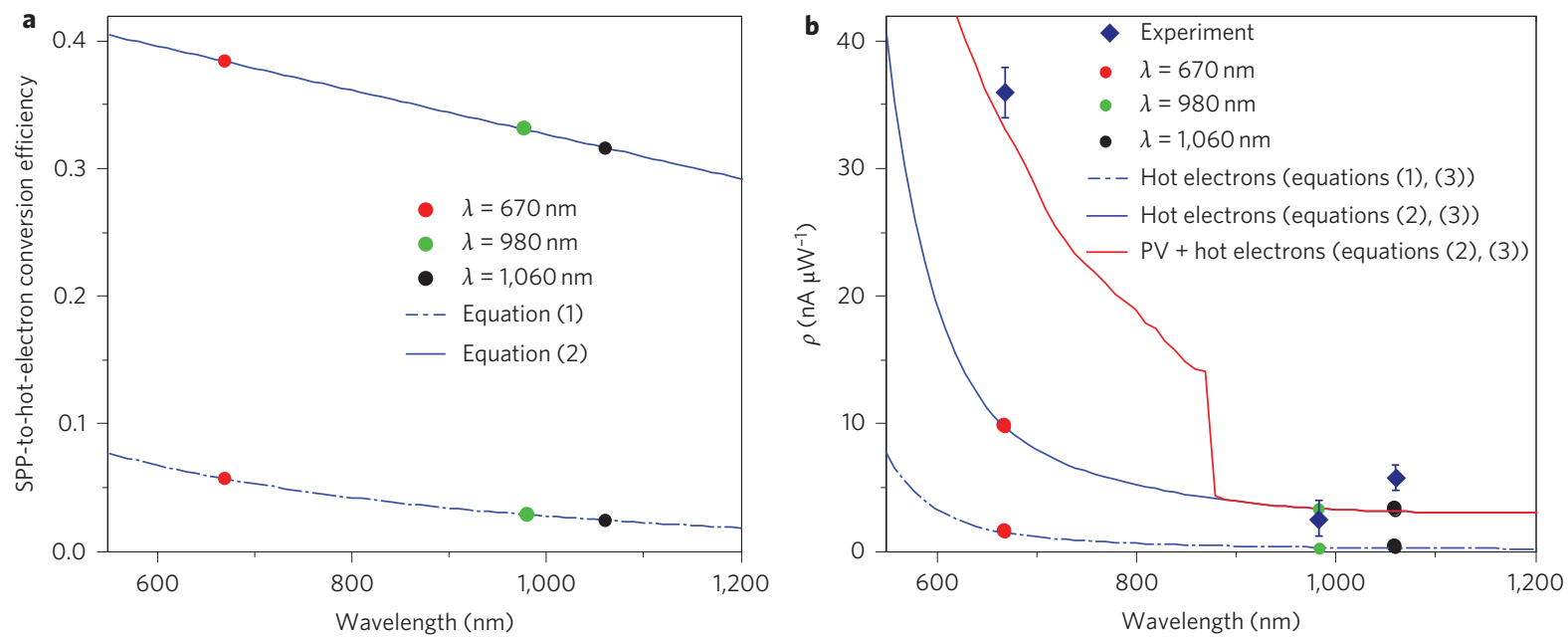

Figure 6 | SPP-to-hot-electron conversion efficiency and responsivity. The electron emission rate occurring at the tip was calculated for a tip radius of $R=25 \mathrm{~nm}$ and SPP optical power of $P=1 \mu \mathrm{W}$ (see numerical calculation in Supplementary Sections 1 and 8 ). a, Conversion efficiency $\eta$ for the $\mathrm{n}$-type GaAs-Au tip Schottky junction as a function of wavelength calculated with equation (1) and equation (2). b, Experimental device responsivity $\rho$ (blue diamonds) and its theoretical determination, equation (3), as a function of wavelength and considering equation (1) (blue dotted line) or equation (2) (blue continuous line). The red line shows the same calculation of equation ( 3 ) + equation (2), adding the photovoltaic (PV) contribution obtained from known literature (Supplementary Sections 7 and 8).

between $\mathrm{Au}$ and GaAs at zero applied bias, $n$ is the ideality factor, $V$ is the applied bias voltage, and $R_{\mathrm{S}}$ is the series resistance.

$\Phi_{\mathrm{B} 0}, n, R_{\mathrm{S}}$ and the onset voltage $V_{\mathrm{on}}$, extracted by fitting the measured $I-V$ characteristic (Supplementary Table 1) with the current equation, reflect the point contact geometry of the tip and the presence of an oxide layer with a thickness of $\sim 0.7 \mathrm{~nm}$ (expected from room-temperature oxidation conditions) ${ }^{36}$.

The breakdown value is around $-5.5 \mathrm{~V}$, about a factor of two less than the corresponding value for a planar macro electrode, expected to be $-12 \mathrm{~V}$ at our doping level. This confirms a nano point contact electrode configuration at the tip junction.

A substantially different behaviour in the $I-V$ curve with laser on and off is found for reverse bias. The inset of Fig. $4 \mathrm{c}$ shows the extra current plot $\left(I_{\text {on }}-I_{\text {off }}\right)$, which agrees with the hot electron current calculated from the theory reported later in this Article. In this case, the level of extra current is consistent with the tip junction geometry and an interfacial oxide layer that gives an extra potential barrier of $\sim 3 \mathrm{~V}$, which bends under reverse bias (Fig. 4a), allowing the extra current injection saturation at about $-4 \mathrm{~V}$. This value is compatible with the hot electron energy generated at $\lambda_{3}$.

Equivalent results for hot electron photocurrent generation were also obtained for the silicon semiconductor, despite its higher surface sensitivity to oxidative environmental conditions (Supplementary Section 11).

\section{Hot-electron nanoscopy}

We exploited SPP-generated hot electron photocurrent in a Schottky diode formed from an adiabatic plasmonic cone as a basis for the new scanning probe imaging technique. The sample morphology and hot electron current map were acquired simultaneously using an AFM combined with the experimental set-up presented in Fig. 1, in a lock-in detection configuration.

To demonstrate the imaging quality at the nanoscale, we acquired high-resolution topography and photocurrent maps (Fig. 5) at $\lambda_{1}$ and $\lambda_{2}$ for two different substrates. In the first case (at $\lambda_{1}$, Fig. $5 \mathrm{a}, \mathrm{c}$ ), the topography/current maps were obtained on GaAs, locally oxidized with a nanometric pattern using a high field discharge technique $^{37,38}$ (as described in Supplementary Section 2), providing a profile thickness of less than $1 \mathrm{~nm}$. The continuous and long-lasting line detection profile demonstrates the stability, reproducibility and control of the scanning probe technique ${ }^{39}$ used in the detection of conductive features of nanometric scale, with an average (unconvolved) spatial resolution below $50 \mathrm{~nm}$ easily recognizable approaching the vertices in the designed pattern. The representative scanning lines (i), (ii) and (iii) in Fig. 5b,d contain all the quantitative metrological information about the linewidth, height and current intensity needed to locally characterize the sample. The second experiment (Fig. 5e,g) was performed at wavelength $\lambda_{2}$ on locally $\mathrm{Ga}$ ion-implanted GaAs. This sample shows an increased conductivity due to $30 \mathrm{keV}$ focused implanted ions. It shows further that imaging by hot electrons can efficiently work with very low contrast topography (Fig. $5 \mathrm{~g}$ ) with both oxide and conductive nanopatterned structures. We observe that the photocurrent map measurements show linewidths slightly larger than the patterned lines. The metrological difference $(\sim 30 \mathrm{~nm})$ is due to the chemical sensitivity of the hot electrons and the effective ion implanted area. In fact, as a result of ion scattering, the linewidths become larger than the directly exposed structures. In Supplementary Section 2 we further present maps of ion-implanted GaAs at a higher dose where it is possible to recognize a non uniform ion implantation process combined with surface ion milling.

We note that the present experimental set-up can be further improved in terms of resolution and photocurrent generation efficiency. In particular, the nanotip can be fabricated with a radius of curvature below $10 \mathrm{~nm}$. This would mean a strong improvement in terms of energy concentration, linear momentum exchange to hot electrons, and further confinement of the depleted volume. Our conservative choice of using a $25 \mathrm{~nm}$ radius of curvature was driven by the aim to provide a convincing and robust proof of concept, and better reproducibility of the experimental results.

\section{Theoretical model}

In Supplementary Section 8 we introduce a specific model to treat hot electrons generation by adiabatic SPPs for the case of a conical geometry. In this case, the transfer efficiency-that is, the probability for a hot electron generated from the SPP decay to be transferred across the Schottky junction from the metal into the semiconductor-is given by a modified Fowler formula ${ }^{40}$

$$
\eta=\frac{\left(h v-e \Phi_{\mathrm{B}}\right)^{2}}{4 E_{\mathrm{F}} h v}
$$


where $E_{\mathrm{F}}$ is the Fermi energy of the metal coating of the tip (SPP taper).

Equation (1) is obtained under the assumption of the conservation of electron linear momentum in the plane of the junction. If such conservation is relaxed by the roughness of the junction, then the probability of the electron transfer is increased significantly. The corresponding expression has the form (Supplementary Section 8)

$$
\eta=\frac{h v-e \Phi_{\mathrm{B}}}{2 h v}
$$

The responsivity $\rho$ of the Schottky junction and the total current $J$ across this junction can be written as

$$
J=\rho P
$$

where $P$ is the optical SPP power. The calculated electron transfer efficiency $\eta$ and responsivity $\rho$ for the case of a gold n-type GaAs substrate as functions of wavelength $\lambda$ (ref. 41) are shown in Fig. 6a,b. We set $e \Phi_{\mathrm{b}}=0.42 \mathrm{eV}$ and $E_{\mathrm{F}}=5.53 \mathrm{eV}$.

In Fig. 6a the calculations for $\lambda_{1}, \lambda_{2}$ and $\lambda_{3}$ refer to the two regimes of momentum exchange of equations (1) and (2). We note that the calculated values of diode responsivity are in qualitative and quantitative agreement with those measured in all three experiments. In the photocurrent experiment (Fig. 3a-c) with $I_{\text {on }}$ for all the wavelengths, $I_{\text {on }}\left(\lambda_{1}\right) \approx 35 \mathrm{nA}, I_{\text {on }}\left(\lambda_{2}\right) \approx 1.5 \mathrm{nA}$ and $I_{\text {on }}\left(\lambda_{3}\right) \approx 5.5 \mathrm{nA}$.

In the $I-V$ experiment, the extra current value measurement (Fig. 4b) gave $I_{\text {extra current }} \approx 3 \mathrm{nA}$. In the nanoimaging experiment (Fig. 5b), $I_{\text {map }}\left(\lambda_{1}\right) \approx 4 \mathrm{nA}$ and $I_{\text {map }}\left(\lambda_{2}\right) \approx 1.5 \mathrm{nA}$. Notice that in this case the imaging was performed to optimize the long-term current and signal stability, and is more suitable for a wider variety of samples.

In Fig. $6 \mathrm{~b}$ we report the pure hot electron contribution to the current when equation (1) (dotted blue line) or equation (2) (solid blue line) is considered in the calculations. The continuous red line is the sum of photovoltaic and hot electron contributions. The former is estimated by a diode model in spherical symmetry, taking into account the strong localization due to the tip (Supplementary Section 7).

\section{Conclusions}

The present study aims to demonstrate, as a proof of concept, the relationship between SPPs and the generation of hot electrons, particularly for surface imaging with local chemical sensitivity. In fact, because of the high level of generated photocurrent (in the range of $\mathrm{nA}$ ) and the intrinsic high spatial resolution of the adiabatic nanocone, this approach allowed us to obtain topographic and photocurrent maps of patterned GaAs samples with both thin oxidized nanostructures and ion-implanted lines.

The present results are important for the integration of SPPs with a conventional AFM set-up, including those operating in a wet environment. By exploiting the efficient local conversion of SPPs to hot electrons (above 30\%) a new generation of nanoimaging technique can be conceived, with intrinsic spatial and chemical resolutions falling in the nanometre range.

We finally note that this nanoscopy is fully compatible with scattering-based spectroscopies such as surface-enhanced Raman spectroscopy and surface-enhanced infrared spectroscopy. The combination of optical and electrical excitation will render SPP conversion of interest in nanoscale investigations.

\section{Methods}

The plasmonic structure was fabricated on a commercial AFM cantilever ( $\mu$-masch CSC38) as reported in Fig. 2 (and Supplementary Fig. 2). The device consists essentially of a grating coupler and a tapered nanocone. The structure is gold-coated and includes a grating coupler designed to optimize SPP generation at the downstream cone. Low-spring-constant cantilevers allow real-time photocurrent measurements across the metal tip-semiconductor junction, preserving the tip profile during scanning on the semiconductor sample, as shown in Fig. 1d.

For SPP generation, the laser light was focused solely on the grating. Figure 2a presents a scanning electron microscope (SEM) image of the complete plasmonic structure, the fabrication of which involved focused ion beam milling ${ }^{27}$, electronbeam-induced deposition ${ }^{28}$ and a gold evaporation process.

In Supplementary Section 11 we report current measurements on silicon in order to further demonstrate hot electron photocurrent generation even with a less cooperative surface (silicon suffers from quick native oxide layer formation). Indeed, from an experimental point of view, the use of silicon is not the best choice for a proof of concept due to its surface sensitivity to environmental conditions. This is one of the considerations that guided us to use a GaAs surface, it being more stable, therefore enabling us to show the role of hot electrons. Beyond these additional difficulties, the results for silicon are compatible with hot electron generation in GaAs.

The $I-V$ characteristics of the tip junction in Fig. 4 were obtained by applying a slow $(0.5 \mathrm{~Hz})$ symmetric sawtooth ramp, with increasing amplitude up to $20 \mathrm{~V}$ (peak-to-peak), and repeating it to obtain temporal characterization of the junction (Supplementary Section 3). All measurements were performed while the laser power was routinely modulated on and off.

To manage unwanted drift effects due to mechanical instabilities, we modulated the laser amplitude with a square wave at non-resonant frequencies, specifically $317 \mathrm{~Hz}$ and $571 \mathrm{~Hz}$, with a duty cycle of $50 \%$. These frequencies represent a quasid.c. modulation compared to the typical thermalization timescale occurring in semiconductors and to the data-sampling frequency $(200 \mathrm{kHz})$. This modulation is still fast enough to overcome the effect of slow drifts and most acoustic excitations typically affecting scanning probe measurements.

We further characterized the GaAs samples with absorption and photoluminescence measurements (reported in Supplementary Section 10). The results demonstrate that the measured photocurrents are not attributable to the excitation of impurity or defect midgap states (even at a longer wavelength of $\lambda=1,060 \mathrm{~nm}$ ), but are due to the conversion of plasmon polaritons to hot electrons.

\section{Received 18 April 2013; accepted 11 September 2013;} published online 20 October 2013

\section{References}

1. Barnes, W. L. Surface plasmon-polariton length scales: a route to sub-wavelength optics. J. Opt. A 8, S87-S93 (2006).

2. Proietti Zaccaria, R. et al. Fully analytical description of adiabatic compression in dissipative polaritonic structures. Phys. Rev. B 86, 035410 (2012).

3. Shen, H., Guillot, N., Rouxel, J., de la Chapelle, M. L. \& Toury, T. Optimized plasmonic nanostructures for improved sensing activities. Opt. Express 20, 21278-21290 (2012).

4. Guillot, N. et al. Surface enhanced Raman scattering optimization of gold nanocylinder arrays: influence of the localized surface plasmon resonance and excitation wavelength. Appl. Phys. Lett. 97, 023113 (2010).

5. Gucciardi, P. G. et al. Light depolarization induced by metallic tips in apertureless near-field optical microscopy and tip-enhanced Raman spectroscopy. Nanotechnology 19, 215702 (2008).

6. D'Andrea, C. et al. Optical nanoantennas for multiband surface-enhanced infrared and Raman spectroscopy. ACS Nano 7, 3522-3531 (2013).

7. Rauhut, N. et al. Antenna-enhanced photocurrent microscopy on single-walled carbon nanotubes at $30 \mathrm{~nm}$ resolution. ACS Nano 6, 6416-6421 (2012).

8. Goykhman, I., Desiatov, B., Khurgin, J., Shappir, J. \& Levy, U. Locally oxidized silicon surface-plasmon Schottky detector for telecom regime. Nano Lett. 11, 2219-2224 (2011)

9. Knight, M. W., Sobhani, H., Nordlander, P. \& Halas, N. J. Photodetection with active optical antennas. Science 332, 702-704 (2011).

10. Goykhman, I., Desiatov, B., Khurgin, J., Shappir, J. \& Levy, U. Waveguide based compact silicon Schottky photodetector with enhanced responsivity in the telecom spectral band. Opt. Express 20, 28594-28602 (2012).

11. Mubeen, S. et al. An autonomous photosynthetic device in which all charge carriers derive from surface plasmons. Nature Nanotech. 8, 247-251 (2013).

12. De Angelis, F. et al. Breaking the diffusion limit with super-hydrophobic delivery of molecules to plasmonic nanofocusing SERS structures. Nature Photon. 5, 683-688 (2011)

13. De Angelis, F. et al. Nanoscale chemical mapping using three-dimensional adiabatic compression of surface plasmon polaritons. Nature Nanotech. 5, 67-72 (2010)

14. Babadjanyan, A. J., Margaryan, N. L. \& Nerkararyan, K. V. Superfocusing of surface polaritons in the conical structure. J. Appl. Phys. 87, 3785-3788 (2000).

15. Stockman, M. I. Nanofocusing of optical energy in tapered plasmonic waveguides. Phys. Rev. Lett. 93, 137404 (2004).

16. Stockman, M. I. Erratum: nanofocusing of optical energy in tapered plasmonic waveguides [Phys. Rev. Lett. 93, 137404 (2004)]. Phys. Rev. Lett. 106, 019901 (2011). 
17. Bao, W. et al. Mapping local charge recombination heterogeneity by multidimensional nanospectroscopic imaging. Science 338, 1317-1321 (2012).

18. Gramotnev, D. K., Vogel, M. W. \& Stockman, M. I. Optimized nonadiabatic nanofocusing of plasmons by tapered metal rods. J. Appl. Phys. 104, 034311 (2008).

19. Aeschlimann, M. in Encyclopedia of Nanoscience and Nanotechnology (ed. Singh Nalwa, H.) Vol. 3, 29-40 (American Scientific, 2004).

20. Hasegawa, K., Nöckel, J. U. \& Deutsch, M. Curvature-induced radiation of surface plasmon polaritons propagating around bends. Phys. Rev. A 75, 063816 (2007).

21. Wiener, A., Fernandez-Dominguez, A. I., Horsfield, A. P., Pendry, J. B. \& Maier, S. A. Nonlocal effects in the nanofocusing performance of plasmonic tips. Nano Lett. 12, 3308-3314 (2012).

22. Melikyan, A. \& Minassian, H. On surface plasmon damping in metallic nanoparticles. Appl. Phys. B 78, 453-455 (2004).

23. Sönnichsen, C. et al. Drastic reduction of plasmon damping in gold nanorods. Phys. Rev. Lett. 88, 077402 (2002).

24. Issa, N. A. \& Guckenberger, R. Fluorescence near metal tips: the roles of energy transfer and surface plasmon polaritons. Opt. Express 15, 12131-12144 (2007)

25. Kats, M. A., Yu, N., Genevet, P., Gaburro, Z. \& Capasso, F. Effect of radiation damping on the spectral response of plasmonic components. Opt. Express 19, 21748-21753 (2011).

26. Bharadwaj, P., Bouhelier, A. \& Novotny, L. Electrical excitation of surface plasmons. Phys. Rev. Lett. 106, 226802 (2011).

27. Cabrini, S. et al. Focused ion beam lithography for two dimensional array structures for photonic applications. Microelectron. Eng. 78-79, 11-15 (2005).

28. De Angelis, F. et al. A hybrid plasmonic-photonic nanodevice for label-free detection of a few molecules. Nano Lett. 8, 2321-2327 (2008).

29. Donolato, C. Approximate analytical solution to the space charge problem in nanosized Schottky diodes. J. Appl. Phys. 95, 2184-2186 (2004).

30. Smit, G. D. J., Rogge, S. \& Klapwijk, T. M. Scaling of nano-Schottky-diodes. Appl. Phys. Lett. 81, 3852-3854 (2002).

31. Sondheimer, E. H. The mean free path of electrons in metals. Adv. Phys. 50, 499-537 (2001)

32. Dalal, V. L. Simple model for internal photoemission J. Appl. Phys. 42, 2274-2279 (1971).

33. Simon, M. \& Sze, K. K. N. Physics of Semiconductor Devices 3rd edn (Wiley-Interscience, 2006).

34. Card, H. C. \& Rhoderick, E. H. Studies of tunnel MOS diodes I. Interface effects in silicon Schottky diodes. J. Phys. D 4, 1589-1601 (1971).

35. Hudait, M. K. \& Krupanidhi, S. B. Doping dependence of the barrier height and ideality factor of $\mathrm{Au} / \mathrm{n}-\mathrm{GaAs}$ Schottky diodes at low temperatures. Physica B 307, 125-137 (2001)
36. Hardikar, S., Hudait, M. K., Modak, P., Krupanidhi, S. B. \& Padha, N. Anomalous current transport in Au/low-doped n-GaAs Schottky barrier diodes at low temperatures. Appl. Phys. A 68, 49-55 (1999).

37. Lorenzoni, M., Giugni, A. \& Torre, B. Oxidative and carbonaceous patterning of Si surface in an organic media by scanning probe lithography. Nano. Res. Lett. 8, 75 (2013)

38. Torrey, J. D. et al. Scanning probe direct-write of germanium nanostructures. Adv. Mater. 22, 4639-4642 (2010).

39. Canale, C., Torre, B., Ricci, D. \& Braga, P. C. Recognizing and avoiding artifacts in atomic force microscopy imaging. Methods Mol. Biol. 736, 31-43 (2011).

40. Fowler, R. H. The analysis of photoelectric sensitivity curves for clean metals at various temperatures. Phys. Rev. 38, 45-56 (1931).

41. Palik, E. D. Handbook of Optical Constants of Solids. Part II (Academic, 1985).

\section{Acknowledgements}

The authors thank M. Lorenzoni for providing the patterned sample for hot-electron nanoimaging. The authors also thank S. Lupi for infrared absorption measurements, B. S. Ooi for helping with $980 \mathrm{~nm}$ measurements, and A. Fratalocchi for several useful discussions. E.D.F. acknowledges support from European Projects Nanoantenna (FP7 No. 241818, FOCUS FP7 No. 270483). M.I.S. acknowledges support from the Max Planck Society and the Deutsche Forschungsgemeinschaft Cluster of Excellence: Munich Center for Advanced Photonics (http://www.munich-photonics.de) and the Chemical Sciences, Biosciences and Geosciences Division (grant no. DE-FG02-01ER15213) of the Materials Sciences and Engineering Division of the Office of Basic Energy Sciences, Office of Science, US Department of Energy (grant no. DE-FG02-11ER46789).

\section{Author contributions}

M.I.S. and E.D.F. conceived the adiabatic plasmonic hot-electron nanoscopy. A.G., A.T., B.T. and E.D.F designed the experiments and A.G., A.T. and B.T. performed the experiments and analysed the data. R.P.Z. and A.A. performed the numerical simulations. M.F. and M.M. realized the nanostructured devices. E.D.F. supervised the whole project. All authors contributed to the discussion of the results and to writing the manuscript.

\section{Additional information}

Supplementary information is available in the online version of the paper. Reprints and permissions information is available online at www.nature.com/reprints. Correspondence and requests for materials should be addressed to E.D.F.

\section{Competing financial interests}

The authors declare no competing financial interests. 